\title{
“Life, Memory, Recognition and Aging” of Grey Tin
}

\author{
A. D. Styrkas \\ Institute of Solid State Physics RAS, Chernogolovka, Russia \\ Email: astyrkas@issp.ac.ru
}

How to cite this paper: Styrkas, A.D. (2016)

"Life, Memory, Recognition and Aging" of Grey Tin. Journal of Materials Science ano Chemical Engineering, 4, 1-11. http://dx.doi.org/10.4236/msce.2016.411001

Received: September 26, 2016 Accepted: November 8, 2016

Published: November 11, 2016

Copyright $\odot 2016$ by author and Scientific Research Publishing Inc. This work is licensed under the Creative Commons Attribution International License (CC BY 4.0).

http://creativecommons.org/licenses/by/4.0/

\begin{abstract}
It was shown that tin has two types of memory: 1) "memory of the structure" about of the event when it was in the $\alpha$ configuration, and 2) "memory of recognition (discern)" whereby tin recognises that an object with which it in contact, was previously in contact with substances of a particular type ("infection"). Transformations of metallic white tin into the grey semiconductor occur with the help of either small pieces of grey tin or other substances isomorphous with grey tin [1] [2] [3]. These pieces (when pressed into white tin) initiate phase transition (by "infection") from white tin into grey tin. Once the tin is transformed into its grey form, it retains a "memory" about this after it is transferred back into white tin. Thus, for second and subsequent phase transformations, there is no need for external initiators to be used. The tin has the "memory of recognition" too-when the tin can recognises that an object with which it is in contact, was previously in contact with the "infection". This phenomenon is concerned with the aging of tin: firstly, with the loss of "memory of the structure" of tin of the event when it was in the grey tin configuration, and, secondly, with the loss of "memory of recognition" of tin whereby the tin recognises that an object with which it is in contact, was previously in contact with substances of a particular type. Factors that effect the aging of tin has been studied in detail and an explanation of the mechanism of action of these factors has been suggested.
\end{abstract}

\section{Keywords}

Tin, Memory, Recognition, Infection, Aging, Loss of Memory

\section{Introduction}

This work is about the study of phenomenons of loss of "memory structure" tin (the memory of the stay in the structure of grey tin) and "memory recognition" ("recognition" that the objects with it comes into contact, or are themselves substances of a certain type, or such were in contact). The paper identified the factors affecting "loss of memory" under "aging" or "resumption" of these types of "memory" and proposed the 
mechanism of these phenomenons.

Tin is known to people more than 7 thousand years. In connection with its location in the center of the Periodic table [1], at a crossroads of changes in the properties of the elements, it has amazing properties. Just treated him like any other lifeless body, accompanied by terms referring to living creatures: "cry", "plague", "infection", "corruption", "disease", "memory", "recognition", and "aging". Many legends and incredible tales are associated with allotropy the transformation of tin ("tin plague"): French in 1812 lost pants because at the cold their tin buttons crumbled in the dust. The organ pipes suddenly collapsed in one of the winters, although experienced many years at the same frost. Tin kettles at the warehouse, ingots of tin in Siberia, pewter spoons, geologists have turned into gray dust; the Antarctic expedition of Robert Scott in 1912, deprived of food and fuel due to the destruction of tin junctions with the same transformation of tin, was tragically killed, etc.

Being a group IV element, an analog of carbon, tin also has a few modifications [1]. Ordinary white tin is stable at normal temperatures, metal with a tetragonal structure (Figure 1) and electronically as s2p2, density $\mathrm{d}=7.32$ and $\mathrm{TM}=232^{\circ} \mathrm{C}$. There is as if in pain while bending screams ("tin cry"). Phase transition to low-temperature modification, aptly named "tin plague" because Sn "ill," of unknown infection, after the "cure" maintaining "disease symptoms", and there is the transformation white to grey structure. $\alpha$-Sn-covalent crystal with the cubic (Figure 2) structure of diamond ( $\mathrm{a}=6.46(\mathrm{~A})$, stable at low temperatures, with the electronic state of sp3, a density of $d=5.8$, a temperature of phase $\beta$ - $\alpha$ balance $+13.2^{\circ} \mathrm{C}$. Electronic and structural adjustment leads to a change in the density.
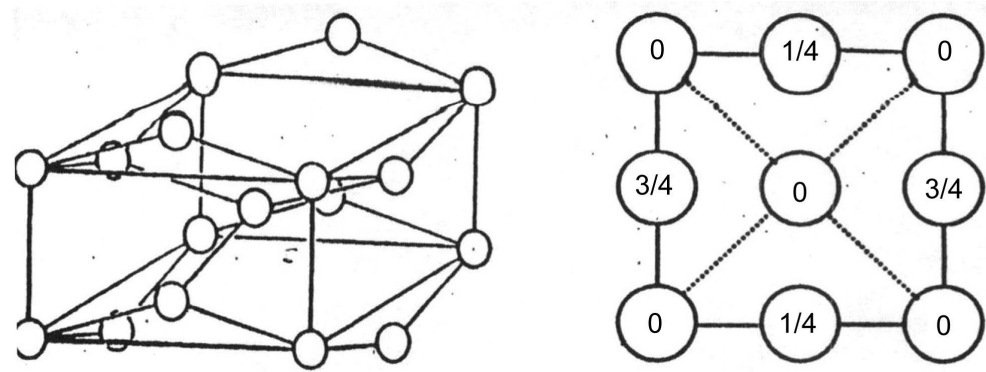

Figure 1. The structure of white tin.
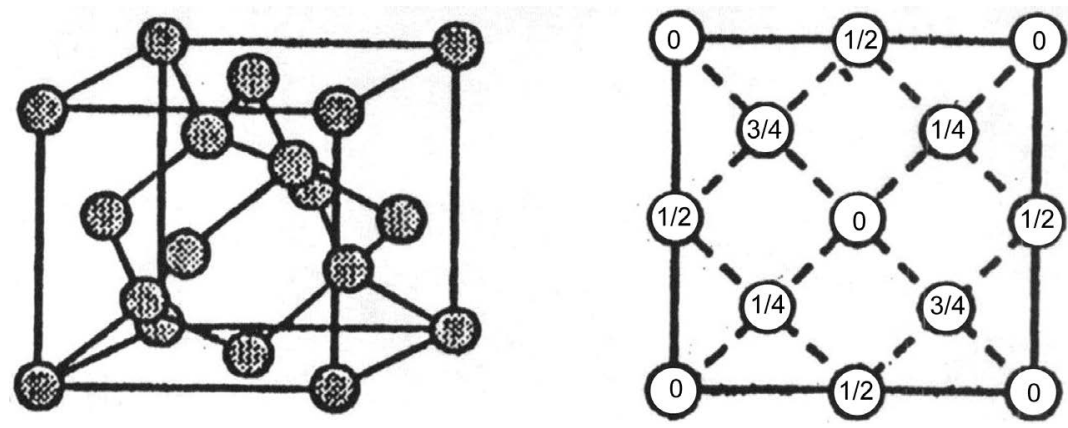

Figure 2. Structure of grey tin. 
Phase transition of $\mathrm{Sn}$ is accompanied by a huge (26.6\%) volume effect, causing the probability of spontaneous generation centers of the new phase is very small. Such a three-dimensional effect and the changing nature of the chemical bonds for ensure the stability of the diamond, is metastable under normal conditions, modification of carbon, the equivalent of tin. Usually, even severe hypothermia (down to liquid He temperatures) is not dangerous for white tin, but the phase transition happens quickly when there is "germ" of "infestation", shiny metal crumbles to a gray powder, what keeps "memory" [2], "corruption" saved forever, traces of "illness" does not disappear even after melting of the metal [3].

\section{Experimental Data and Discussion}

Studied the tin of high purity brand ovch-0000 (99.9999\%) Samples of tin were prepared in the form of smooth plates of laminated and annealed in vacuum of metal. For infection used the flat polished plates of monocrystalline InSb and CdTe. In the freezer withstands temperature of $258 \mathrm{~K}$. To study the influence of the atmosphere, the samples were placed in vials, which created a vacuum $10^{-6}-10^{-7} \mathrm{PA}$ then was filled with gas. Ampoule to study the effect of ozone was made of quartz. Irradiation was carried out by UV from the mercury lamp. Each of the experiments was made on a series of samples of 25; control samples of each type of monitoring were not less than 10 pieces.

\subsection{The Memory Structure and Aging}

The process of phase transformation of tin is rather complicated and depends on many parameters [4] [5] [6] [7] [8]. Transition is always necessary sources of infection, which can be gray tin [1] [2] [3] [4] (isoelectronic counterparts InSb and CdTe [4], the metastable [9] ice cube [7] [10]). Without priming transferring the tin into the grey modification is impossible, contrary to the opinion of even such an authority as Folmer [11], who believes that inanimate nature is possible and "spontaneous". However, on the basis of a detailed study of the mechanism of allotropy tin can be argued: spontaneous generation never occurs in any hypothermia (paracymene, deviations from equilibrium), and all the apparent instances of its manifestation due to introduction of a germ from outside, and nature of subsequent growth phase is due to the number of embryos and speed of the process at least in the solid body. White tin is easily "tainted" by introducing into it the seed, which is similar to $\alpha$-Sn. InSb and CdTe [4] with him in a similar type of chemical bond and structure, but also the cell parameters (different parameter but only on the share \%). When $\beta$-Sn to incorporate seed to a $\alpha$-Sn, like crystal immersed in a supersaturated solution or supercooled melt begins the growth of a new phase on the faces of the crystal related structures. Without the seed Sn is stored for years even at the temperature of maximum rate of transformation of pure tin

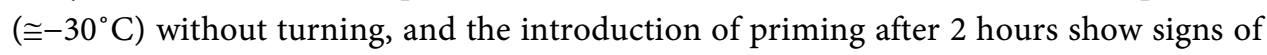
infection, and soon the entire crystal (like the plague!) is in $\alpha$-able. The transition back to $\beta$ phase occurs just after heating. After this, the metal appears to be "corrupted" even after melting it slightly different density and has a dark color, less shiny than the original tin. There are some irreversible changes-there is a certain amount of SnO. It turned out that the tin "remembers" that it was in $\alpha$-structure, and re-transfer to it of 
tin, translated in white $\beta$-modification, at lower temperatures occurs not only spontaneously, without priming, but much faster [2]. From the seed first darkening and beginning of growth "bubo" become visible after 2 hours, and reconfiguration of the entire sample fully completes in less than 15 minutes. "Memory structures" used for preparation of Sn powders of high purity [12] [13] [14] [15] by repeated transfer from $\beta$ - $\alpha$ phase and back. Volume effect leads to self-destruction tin without external influences, which allows to preserve high purity of the powder [14] [15] for all impurities except oxygen. Powders of Sn especially high purity, necessary for accurate solder, especially when you have to enter them into very fine products, they are valuable as anti-friction materials as effective catalysts with a superior working surface for organic syntheses, for precision synthesis of trace amounts of compounds of a given composition containing the tin (Figure 3 ).

However, the memory over time, gradually lost, and her loss is different depending on the day. In a vacuum it lost noticeably after the 15th cycle (Figure 3, curve 1), in the atmosphere of inert gases and in the presence of water vapor it lasts longer (Figure 3, 2-5), in air it does not disappear (Figure 3,6), within a few years of research remained almost without damage. With time, memory fades and is lost [16]. Speed of memory loss increases with temperature and depends on atmosphere (Figure 4).

(Figure 4, curves 1 and 3) memory in the aging in vacuum for 8 hours lost is much stronger than in air. Memory sustained in the air samples can be partly restored by treatment with ozone in a moist atmosphere (curve 6), or electrochemical. Anodic oxidation (curve 4) and activation of AC (curve 5) partially restore memory. Cathodic polarization also promotes acceleration of its losses (curve 2).

Memory tin that is gray, disappears in vacuum and in an oxygen-free medium. The rate of aging depends on ability of tin to oxidize. "Memory structure" does not occur in the samples undergone $\beta-\rightarrow \alpha \rightarrow \beta$ switching in vacuum and even in the atmosphere of water vapor in the absence of oxygen. So it would be natural to assume that memory of this kind is responsible, some oxides. Ordinary oxide film, ever-present on the surface

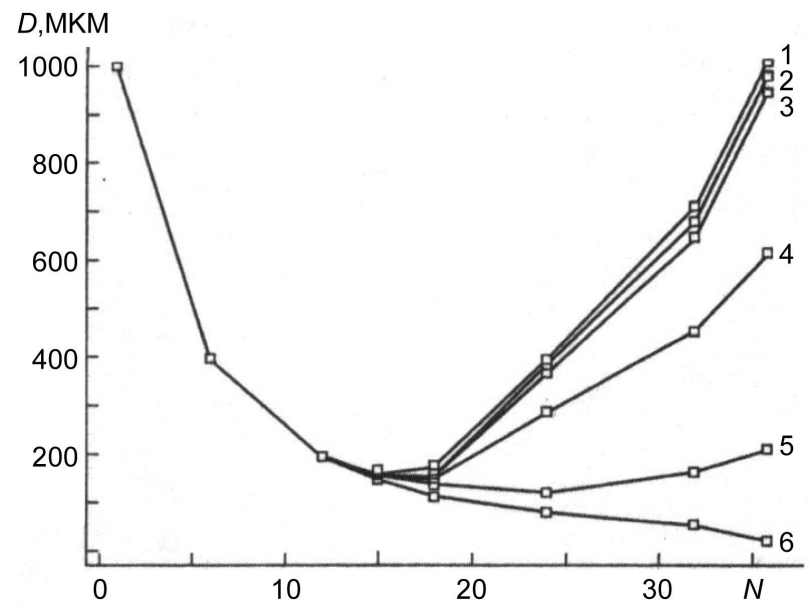

Figure 3. Particle size (D) of the number phase transitions of tin (N) under different atmosphere. 1-vacuum; 2-dry argon; 3-dry nitrogen; 4-dry carbon dioxide; 5-water vapor; 6-air atmosphere". 


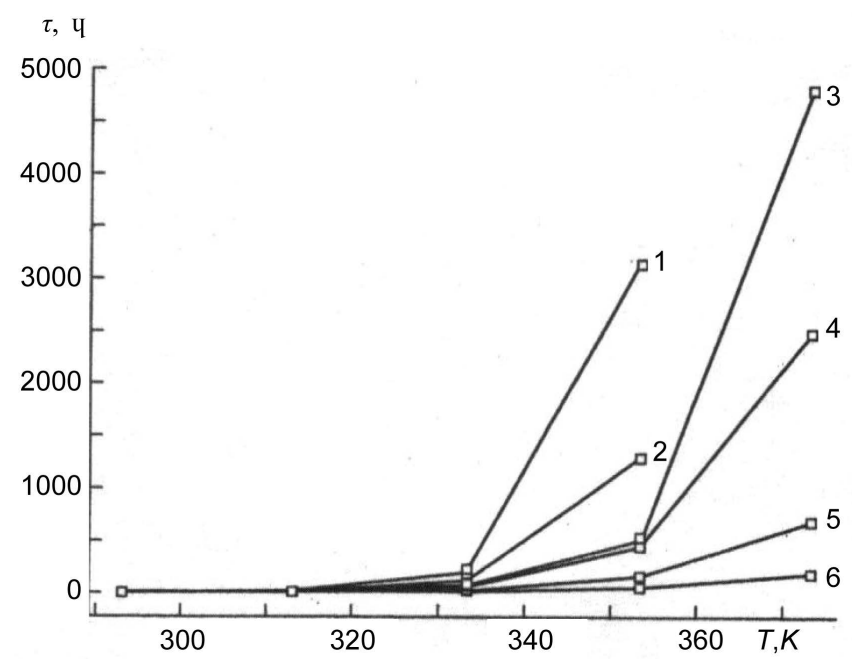

Figure 4. "The dependence of induction period (hours) "memories" by $\mathrm{Sn}$ particles from temperature $(\mathrm{T})$ of exposure in white phase at re-switching them to grey-phase under different atmosphere and electrode polarization. 1-vacuum; 2-cathodic polarization; 3-common; 4-anode polarization; 5-alternating-current polarization; 6aging in a humid atmosphere with ozone."

of white tin that protects the metal from further oxidation, does not affect the memory, will never catalyze the transition. Obviously, the decisive factor is not her, but the oxide which forms only on the surface of the grey tin. To test assumptions sample, tin with the seed was placed in vacuum and cooled to complete the transition of white tin to grey, and then was kept for $1-2$ weeks at room temperature to guarantee transition $\alpha$-Sn to $\beta$-phase. Then ampoule was opened and separated part of the powder that was in contact with priming, then Sn powder, were placed in the cold. None of the samples passed into $\alpha$-phase because of the oxidation of exposed white tin. All control samples passed to $\alpha$ phase through the usual 10 - 15 minutes. $\alpha$ Sn, despite of low temperature, oxidized considerably stronger than $\beta$ Sn. So powder samples in air 50 cycles $\beta \rightarrow \alpha \rightarrow$ $\beta$ transformations, contains, according to gas analysis to 0.23 mass $\%$ of $\mathrm{O}$. There is a metastable $\mathrm{SnO}$ with structure of type $\mathrm{PbO}$ (red litharge), in some respects, akin to structure of the diamond. So this oxide for phase transition is seed, and that it is associated with "memory structure". It epitaxially grows on $\alpha \mathrm{Sn}$ and the transition of $\alpha-\beta$, and when at a temperature above $+12.4 \mathrm{C}$ itself $\alpha \mathrm{Sn}$ ceases to exist, this oxide still exists, and therefore when re-cooling already on it as related to the seed is formed $\alpha$-phase. The oxide is metastable and decays by the reaction of disproportionation to dioxide of tin (IV) and tin according to reaction (1):

$$
2 \mathrm{SnO}=\mathrm{SnO}_{2}+\mathrm{Sn}
$$

But if the tin in $\alpha$ structure has the ability to oxidize by air, it resumed partial loss of oxide(II), i.e. the "memory structure". When transformation were produced in a vacuum without replenishment of the oxide, after 15 cycles, the transition can't happen more. Sn was losing her memory. Powder did not dispersed and become larger due to 
the outbreak of the sticking of the particles causing visible differences between the data sieve and microscopic (Figure 5) analyses of particle size distribution of the particles. Microscopic analysis showed that particles are composed of smaller but stucked together. This phenomenon is found their application for creation tin filters with desired pore sizes, as well as to create catalysts for organic syntheses, in some cases more than powders.

Insights on the mechanism of the processes are in good agreement with the observed results that find a natural interpretation. At high temperatures, metastable oxide active disproportionate on (1), and is oxidized to $\mathrm{Sn}(\mathrm{IV})$ on (2):

$$
2 \mathrm{SnO}+\mathrm{O}_{2}=2 \mathrm{SnO}_{2}
$$

For a similar reason, the memory of your stay in $\alpha$-structure of tin in the smelting process is completely lost. After melting the remaining stable oxides remain on the appearance metal of the consequences of the disease are called "corruption" of the metal.

Even Mendeleev said: "if tin grind and pour a solution of tin in $\mathrm{HCl}$, then the transition tin of plain grey done relatively soon $\hat{i}$ [1]. This is the effect of the different solvents that are currently widely known. It is believed that the reason for the acceleration of the transition by the action of solvents can be remove surface oxide kayer, which is to transform useless, however, the nature of the action caused by this source of infection is not revealed. In the same way explained and the acceleration of phase transformation during mechanical or radiative disturbances of its integrity, but such violation does not always lead to infection. It seems more likely that under the action of $\mathrm{HCl}$ on the tin compounds are formed Sn(II), which upon hydrolysis give active tin oxide(II) according to R. (3) and (4):

$$
\begin{gathered}
\mathrm{Sn}+2 \mathrm{HCl}=\mathrm{SnCl}_{2}+\mathrm{H}_{2} \\
\mathrm{SnCl}_{2}+\mathrm{H}_{2} \mathrm{O}=\mathrm{SnO}_{2}+2 \mathrm{HCl} .
\end{gathered}
$$

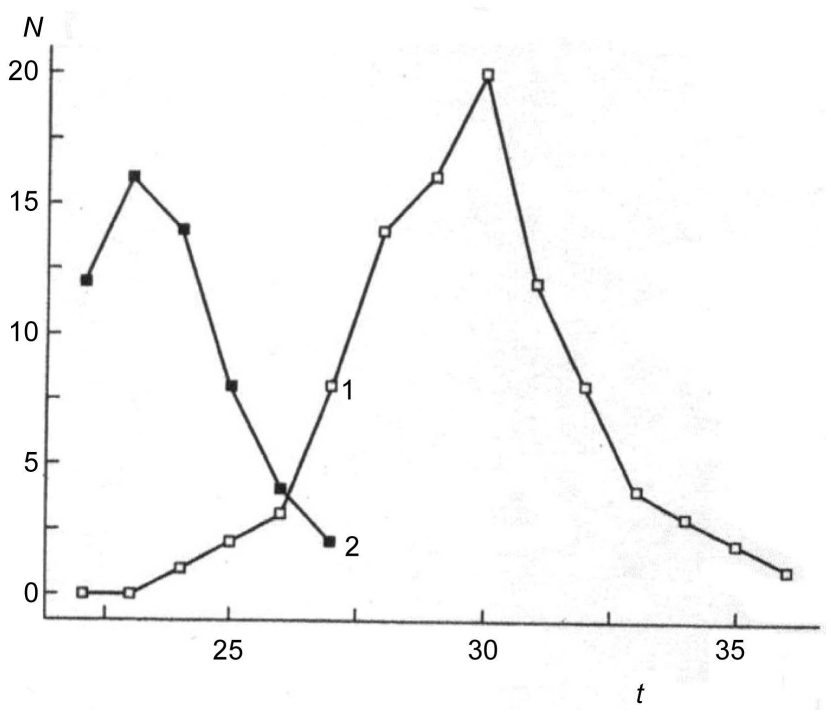

Figure 5. The dependence of the number of converted samples of tin $(N)$ versus time (hours). 


\subsection{Recognition Memory and Aging}

In order for the crystal to grow on the seed, the required molecular should be contact of seed and nurseries. For liquids (solutions, melts), it is easily achievable. In a solid that is more difficult to achieve. This is achieved by rubbing, injecting the embryo "infection" [4]. However, it turned out that quite a simple contact [2] [3] [4], but the phase transition of white tin to grey enough even the presence of the seed at distances many orders of magnitude greater than intermolecular [5] [6]. 80 samples of tin were put polished monocrystalline plate InSb coated with a polymer film thickness of $1 \mu \mathrm{m}$, which excluded the possibility of contact of the tin with the seed, however, in the film photolithographically was made of the window. All 80 samples were infected in a period of about one month. The tin has not crumbled into powder, while remaining in one piece, although increased in size. All samples of gray tin were coarse-crystalline, consisting of only several blocks, or even monocrystalline. All 10 samples that are directly in contact with the InSb turned into powder within a day, at the same time, the same 10 samples, put on plate InSb covered with a continuous polymer film without windows, "not contracted" in the course of the year, after which observation was stopped. Note, however, that even with direct contact with InSb tin was not "infected", if in the environment of water vapor were absent [10].

White tin at low temperatures remains in its metastable state very long. The process of restructuring in a solid is difficult. But it should appear to be only one center of crystallization, as the process of transition is unstoppable. Indentation of the seed provides atomic contact, and the new begins to grow. But famous in the history of the facts of spontaneous infections no one pressed in the seed products. Our experiments proved the possibility of infection at a distance, and showed that responsible water vapor present in the atmosphere. Plate of white tin and InSb, treated with alcohol or acetone for removal of adsorbed water vapor from the atmosphere put on top of each other and tightly pressed to each other by clips ("crocodiles"). 25 such pairs were placed in evacuated ampoules; 25 such pairs were placed in vials with alcohol and acetone, 25 such as "sandwiches" in the desiccator with air (dry), and 10 control pairs just air put in the fridge. None of the 100 samples contracted for the year, while the control properly in the air were caught in 1 - 3 hours. Test specimens removed from the vials and the desiccator in a year, and after 2 days all the samples were converted to $\alpha$-phase.

The tin is manifested, and has another interesting property: it becomes infected upon contact with the inert body, if it was previously in contact with one of those substances that serves as primer [3] [7] [17]. On a clean glass plate at 14 days put polished InSb plate in the freezer. Then samples of InSb were removed and in their place were put plates of white tin. Of the 56 samples of tin in a period of about 25 days contracted 55 . A similar experience was carried out when exposed "infection" on the glass at room temperature, and the result was the same. Then, to elucidate the role of water vapor in the atmosphere taken control 6 parties tin of 10 pieces. All of them were put on the glass that was in contact with the seed within 2 weeks. But before it put the samples, Sn, glasses treated: 1-alcohol; 2-glycerol; 3-water; 4 by heating at about $100 \mathrm{C}$ for 1 hour. 
Glass of 5th party contacted with the InSb in a dry desiccator, and the 6th party had not been in contact with InSb. Of the 60 control samples, none within 6 months of observations is not infected. The memory of "knowing" about contact with seed inert body is not generated in the absence of water vapor, and that which is created, can be destroyed by solvents, solvent, water (ice) or warm-up prior to its evaporation. The memory of this type is also lost during aging and depends on the atmosphere. Figure 6 is visible to see the dependence of "capacity for memory" $\mathrm{R}=\tau$ about $\backslash \tau$ (reciprocal relationship of induction periods in the initial moment after turning $\alpha$ - $\beta$-phase and after “aging”.)

The transfer of information about the structure in the distance [5] [6], and the observed effect [3] [7] [8] [10] infection is not from the seed, and from the subject, who was in contact with her, we call "memory recognition".

There is a modification of the water-metastable cubic ice and the structure of diamond-like (oxygen ions located in the lattice at the diamond law) and the unit cell parameters are very close to gray tin $(\mathrm{a}=6.36 \mathrm{~A})$ [9]. Although water vapor during the deposition on an inert substrate is stable in the structure of the $I_{c}$ at very low temperatures [9] (below about $-120^{\circ} \mathrm{C}$ ), isomorphic crystallization on a substrate through epitaxy takes place at temperatures considerably higher. Cubic ice (or that has significant differences, the so-called "structured water" [18]) grows on a seed, it reaches for the tin and, penetrating into microscale oxide film, performs with white tin molecular contact similar to that which occurs with the introduction of the seed by rubbing, injecting, etc. All the facts of loss of "memory recognition" in the "aging" well within the scope of these ideas. Any factor that prevents the growth of epitaxial cubic ice and its contact with the tin and protect the tin from contagion. Wiping the surface of the inert substance in glycerin, acetone, glycol, alcohol, water, other water miscible solvents prevents plague, dissolving the formed $\mathrm{I}_{\mathrm{c}}$ structures. Any impact on $\mathrm{Sn}$, in violation of the inte-

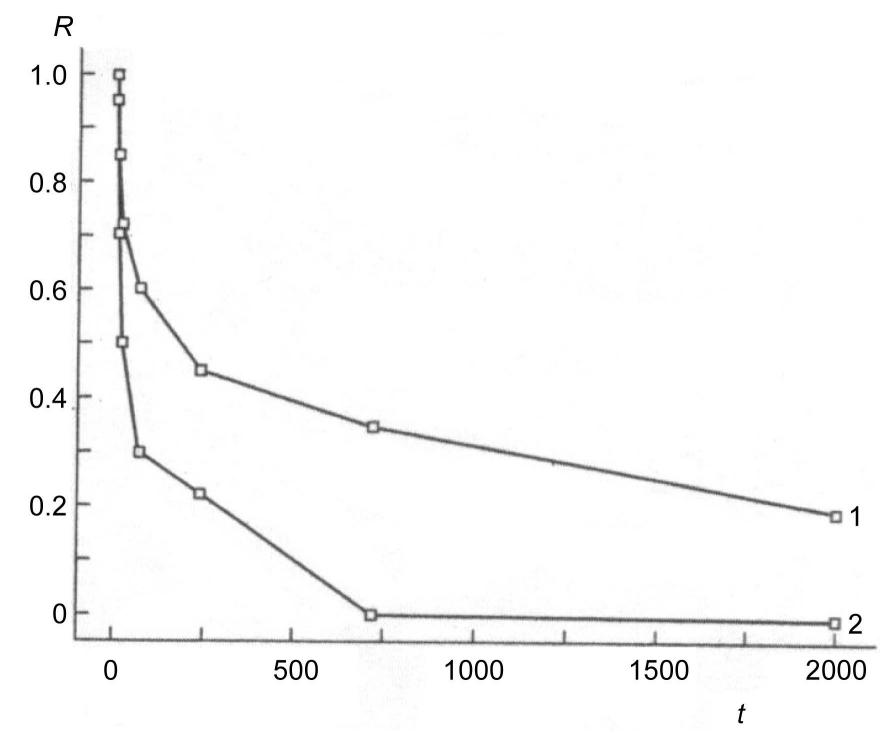

Figure 6. The dependence of the "capacity for memory" ( $\tau$ about $\backslash \tau$ ) on the contact of the inert substances (glass) with "infection" from time (hours) of stay in the normal (1) dry (2) atmosphere. 
grity of the protective surface oxide, contributing to the contact of tin with cubic ice, of course, accelerates the process of "recognition", increasing the chance of infection and reducing the incubation period of a phase transition.

Formed on the seed germ epitaxiale structured water, which is stable even at room and higher temperatures in a thin layer responsible for transmission of infection from inert substances. Loss of memory in aging and accelerate aging factors are also in agreement with the hypothesis of the role of water. Cubic ice can help not only iso-structural materials, but with inert materials. Epitaxial growing on an isostructural substrate, the cubic ice by contact with an inert substance it may remain, and, in turn, serve as a seed for further growth $I_{c}$ (structured water), which causes the infection as soon as it find the contact point with $\beta$-Sn. Therefore, glass and other inert materials $(\mathrm{Cu}, \mathrm{Ni}, \mathrm{W}, \mathrm{Mo}, \mathrm{Si}$, Ge, quartz, marble, getinaks, Plexiglas) which have been in contact with the InSb have mentioned that white tin in contact with him in an atmosphere containing water vapor. Activity glass above, if the surface has defects (roughness, injections, scratches, etc.), because the ice (water) is withheld and retained better than on smooth glass.

It is interesting to note that it seems unexpected in light of the above: the fact of the possibility of contamination of the tin under water! It was not only possible, but possible to obtain not only large crystals [19], and single crystals of a given configuration [20]. Apparently, the semiconductor properties are unique $\alpha$-Sn in the crystalline state, and even of a given shape, which will find new applications in technology.

We recall the experiences of the British to improve the atmosphere on the foggy Albion. They were shooting from the guns charges in order to create small crystals of AgI in the atmosphere. And iodine, and silver are, like the tin with its counterparts in the $\mathrm{V}$ period of mendeleevs table, and AgI also has structure of sphalerite with close to $\mathrm{I}_{\mathrm{c}}$ parameters. Crystals are suitable for creating clouds in the centers of water vapor condensation and depositing them in the form of precipitation from the atmosphere. Becomes clear use for these purposes it AgI. Related to the structure of growing epitaxial layers of water (ice) with the structure $I_{c}$ With further growth phase of water could not remain in a metastable state, and the land falls precipitation is already going to the stable structure of ice, but the role of the seed for their education and growth of the couple played the AgI crystals related with the cubic ice structure.

In conclusion, it should be noted that all the arguments were conducted in view of the possibility of the existence of fairly stable and metastable structures. This phenomenon often occurs naturally, e.g. in Mineralogy, and with the growth of artificial crystals. So, with almost identical short-range order in structures of $\mathrm{ZnS}$ resistant metastable sphalerite and wurtzite in nature is formed predominantly wurtzite [21]. When growing diamond is used, the methods of education in a stable conditions of high pressure [21] with the subsequent preservation due to the kinetic difficulties of the transition in graphite, and epitaxial growth at low pressures, where diamond is metastable [22].

\section{Insights}

For the existence of "memory structure", responsible lower metastable tin oxide can 
form on epitaxiale $\alpha$-Sn. Aging and memory loss associated with its elimination and revival-with the reproduction. "Memory recognition" due to the presence in the atmosphere of water vapor, forming epitaxially on basic structures are isostructural $I_{c}$ which can survive up to high temperatures, while the structure Is under normal conditions unstable already at $-120^{\circ} \mathrm{C}$. Epitaxially formed under the influence of priming, it is able to exist on inert substrates. Aging and the loss of "memory recognition" associated with the removal of structured water. Understanding of the mechanism allows processes to decide and purposefully implement a range of technical tasks, ranging from material science to even ways of dealing with bad weather.

\section{References}

[1] Mendeleev, D. (1934) Bases of Chemistry. ONTI, 2, 707.

[2] Styrkas, A.D. (2004) The Mechanism of the Allotropic Transformation of Tin as a Model of the Mechanism of Biological Processes. Physics of Alive, 1, 82-94.

[3] Aptekar, I.L. and Styrkas, A.D. (1993) Inert Substrate Like Vectors Tin Plague from InSb to $\beta$-Sn. High-Purity Substances, 3, 56-60.

[4] Styrkas, A.D. (2003) Mechanisms Allotropic Transformation of Tin. Inorganic Materials, 39, 806-810.

[5] Styrkas, A.D. (1996) The Answer to the "Tin Plague”. Nature, 11, 33-48. (In Russian)

[6] Aptekar, I.L. and Styrkas, D.A. (1982) Contamination with Tin Plague. DAN USSR, 265, 1155-1157.

[7] Aptekar, I.L. and Styrkas, A.D. (1992) The Nature of "Memory" in the Tin. Dokl AN USSR, $325,80-83$.

[8] Yaroshevskii, A.G., Styrkas, A.D. and Niselson, L.A. (1987) The Influence of the Nature of the Chemical Bond by the Amount of the Distribution Coefficient during Crystallization from the Melt.

[9] Hobbs, P.V. (1974) Ice Physics. Clarendon Press, Oxford, 352.

[10] Aptekar and Styrkas, A.D. (1987) $\mathrm{H}_{2} \mathrm{O}$-Tin Causative Agent of Plague. Dokl AN SSSR, 297, 1149-1151.

[11] Folmer, M. (1986) Kinetics of New Phase Formation. Nauka, Moscow, 208 p.

[12] Styrkas, A.D. and Oganyan, R.A. (1987) High-Purity Tin Powders. Soviet Powder Metallurgy and Metal Ceramics, 26, 867-869. http://dx.doi.org/10.1007/bf00794098

[13] Styrkas, A.D. and Oganyan, R.A. (1989) Preparation and Properties of High-Purity Tin Powders with Large Specific Surface. High-purity Substances, 2, 155-158.

[14] Styrkas, A.D. and Oganyan, R.A. (1992) Production and Properties of Tin Powders Produced by Ultrasonic Atomisation and Solid State Transformation. Powder Metallurgy, 35, 117-119. http://dx.doi.org/10.1179/pom.1992.35.2.117

[15] Styrkas, A.D. and Styrkas, D. (1995) Production of Fine Tin and Lead Powders and Porous Filters. Powder Technology, 84, 35-38. http://dx.doi.org/10.1016/0032-5910(95)02972-5

[16] Styrkas, A.D. (1999) The Loss of "Memory" in the Tin in the "Aging". Dokl AN USSR, 368, 208-212.

[17] Aptekar, I.L. and Styrkas, D.A. (1989) The Influence of Atmospheric Moisture on Polymorphous Transformation of Tin. High-Purity Substances, 2, 21-28.

[18] Styrkas, A.D. (2003) Sn Infection in the Liquid $\mathrm{H}_{2}$ O. Inorganic Materials, 39, 802-805. 
[19] Styrkas, A.D. (2003) Growth of Gray Tin Crystals. Inorganic Materials, 39, 808-811.

[20] Styrkas, A.D. (2005) Preparation of Shaped Gray Tin Crystals. Inorganic Materials, 41, 671675.

[21] Kurdyumov, A.V. and Pilyankevich, A.N. (1979) Phase Transformations in Carbon and Boron Nitride. Naukova Dumka, Kyiv, 188.

[22] Fedoseev, D.V., Deryagin, B.V., Varshavskaya, I.G. and Semenov-Tian-Shansky, A.S. (1984) Crystallization of Diamond. Nauka, Moscow, 136.

Submit or recommend next manuscript to SCIRP and we will provide best service for you:

Accepting pre-submission inquiries through Email, Facebook, LinkedIn, Twitter, etc. A wide selection of journals (inclusive of 9 subjects, more than 200 journals)

Providing 24-hour high-quality service

User-friendly online submission system

Fair and swift peer-review system

Efficient typesetting and proofreading procedure

Display of the result of downloads and visits, as well as the number of cited articles Maximum dissemination of your research work

Submit your manuscript at: http://papersubmission.scirp.org/

Or contact msce@scirp.org 